\title{
Nuevos modelos educativos: los MOOCs como paradigma de la formación online
}

\author{
Raquel Vinader SEgura \\ Universidad Complutense de Madrid \\ rvinader@ucm.es \\ Natalia ABUín VENCES \\ Universidad Complutense de Madrid \\ nabuinve@ccinf.ucm.es
}

\begin{abstract}
Resumen
Un nuevo modelo de sociedad requiere un nuevo modelo de enseñanza. La implantación de las Tecnologías de la Información y la Comunicación (TIC) en la formación abren un nuevo horizonte para educadores y alumnos. Asistimos recientemente a la eclosión de los denominados MOOCS (Massive Online Open Courses): cursos online, gratuitos, sin límite de alumnos y sin requisitos de admisión.

En este trabajo se analiza este nuevo tipo de cursos: desde sus modalidades y las plataformas a través de las que se imparten, hasta los diferentes modelos de negocio que se están planteando para hacerlos rentables.
\end{abstract}

Palabras clave: innovación educativa, e-learning, MOOCs.

\section{New educational models: the MOOCs as online teaching paradigm}

\begin{abstract}
A new model of society requires a new teaching model. The implementation of Information and Communication Technologies (ICTs) in education opens up a new horizon for educators and students. We are attending at the emergence of so-called MOOCs (Massive Open Online Courses): online free courses with an unlimited number of students and without admission requirements.

In this paper we analyze these courses: from the models and platforms through which they are taught, to the different business models that are being considered to make them profitable.
\end{abstract}

Key Words: educational innovation, e-learning, MOOCs

\section{Referencia normalizada:}

Vinader Segura, R. y Abuín Vences, N. (2013) Nuevos modelos educativos: los MOOCs como paradigma de la formación online. Historia y Comunicación Social. Vol. 18. № Especial Noviembre. Págs. 801-814.

Sumario: 1. Introducción. 2. ¿Qué son los MOOCs? 2.1. Fortalezas y debilidades como herramienta educativa. 3. La revolución de la enseñanza superior. 3.1. Udacity. 3.2. edX. 3.3. Coursera. 4.Otras iniciativas: Europa y España. 5. ¿Un nuevo modelo de negocio? 6. Conclusiones. 7. Bibliografía 


\section{Introducción}

Los MOOCs se empezaron a popularizar en 2011, momento en el que numerosas entidades formativas se unieron para crear plataformas de formación abierta en lo que es ya un fenómeno imparable. Udacity, Coursera, Edx o Miriadax son los nombres de algunos de los proyectos más interesantes impulsados por universidades como Stanford, Yale, Princeton, Michigan, Harvard o el Instituto tecnológico de Masachusets (MIT).

Es importante señalar que no todos los MOOCS son iguales. De hecho, no existe unanimidad a la hora de afrontar su estudio. Para algunos autores estos cursos no son ni abiertos, ni masivos ni gratuitos (Zapata-Ros, 2013). Según Lisa Lane (2012) existen tres tipos de MOOCs: basados en el trabajo en red, en tareas o en contenidos. Pero una clasificación popular es la que divide estos cursos atendiendo a la teoría o corriente de aprendizaje correspondiente. Así, puede hablarse de cMOOC, para referirse a los conectivistas, y de XMOOC para los no conectivistas (López, 2012).

Los defensores de los MOOCs afirman que este tipo de cursos llegan para revolucionar uno de los pocos sectores que no ha incrementado su productividad desde sus orígenes: el educativo.

De hecho, si nos remontásemos al siglo XVIII y analizásemos cada uno de los sectores de la sociedad desde la Revolución Industrial, nos daríamos cuenta de que la educación es uno de los pocos que no ha incrementado su productividad, a pesar de que la tecnología así lo permite. Los avances han permitido que las fábricas produzcan cada vez más y con el empleo de menos tiempo y esfuerzo gracias a las máquinas. Sin embargo, el sector educativo sigue empleando las mismas técnicas de enseñanza que se empleaban en la Grecia Clásica: clases magistrales, exámenes y evaluaciones.

No es que la tecnología no haya avanzado en el sector educativo, al contrario. Tras la Sociedad Industrial ha llegado la Sociedad de la Información, y también la denominada del Conocimiento. La Sociedad de la Información hace referencia a la mayor capacidad tecnológica para almacenar información y hacerla circular más rápidamente y con mayor capacidad de difusión. Mientras que, la Sociedad del Conocimiento se refiere a la apropiación crítica y selectiva de la información protagonizada por ciudadanos que saben cómo aprovecharla. En este entorno con tanta capacidad de comunicación y tanta información en circulación ¿se ha incrementado la productividad en la educación? De momento hemos podido observar que a parte del uso del correo electrónico, la educación superior sigue las mismas rutinas que hace siglos.

Actualmente, inmersos en pleno proceso de implantación del Espacio Europeo de Educación Superior, y con nuestro país inmerso en una profunda crisis económica, es un hecho que hay cada vez más alumnos y menos profesores . Cabe preguntarse entonces si las TIC podrían ayudarnos a atravesar esta etapa de profundos cambios mejorar los resultados del sistema educativo: satisfacer las necesidades de conocimiento de más alumnos consumiendo menos esfuerzo y con escasos recursos económicos. 
La respuesta puede estar en los denominados, cursos online, gratuitos, sin límite de alumnos y dirigidos por los profesores más prestigiosos del mundo.

\section{2. ¿Qué son los moocs?}

Los Massive Online Open Courses (MOOCs) alcanzaron su popularidad en 2011, aunque la idea empezó a gestarse casi una década antes. Tienen su origen en el concepto de Social Learning que hace referencia a la posibilidad de aprender través de las interacciones con otros. Según Atkins et alt (2007) las tecnologías Web desarrolladas en las dos últimas décadas han provocado que la oferta educativa a través de la red se haya incrementado notablemente. La Web 2.0 permite a los usuarios interactuar con la información y el conocimiento de una manera más activa en una gran variedad de formatos y actividades educativas (Seely, 2008).

En este entorno social donde los internautas quieren ser los protagonistas y compartir información y conocimientos surgen los MOOCs, cuyo germen fueron las plataformas Fhatom y AllLearn.

Fathom fue un portal de aprendizaje online creado en el año 2000 por la Universidad de Columbia. Este portal ofrecía cursos de Columbia y de otras instituciones académicas a cambio de una tasa. Esta plataforma llegó a tener 65.000 usuarios en más de 50 países. Sin embargo las tasas de matrícula que se cobraban a los estudiantes no llegaban para cubrir los costes de producción de los materiales por lo que cerró en 2003 .

Por su parte, AllLearn fue una plataforma creada por las universidades de Stanford, Yale y Oxford en 2001. Inicialmente ofrecía cursos sólo para alumnos de estas universidades, pero posteriormente se permitió el acceso a usuarios ajenos a estas instituciones. En este caso los cursos tenían un valor de 200 dólares, pero el modelo de negocio también fracasó y en 2006 la plataforma cerró sus puertas virtuales.

Este modelo educativo volvió a emerger en 2008 cuando Cormier y Alexander acuñaron el concepto de Masive Online Open Course (MOOC) para referirse a un experimento pedagógico centrado en crear un entorno de aprendizaje más conectado y democrático. Este experimento fue el curso Connectivism and Connective Knowledge y dirigido por George Siemens de la Athabasca University y Stephen Downes del National Research Council. En este curso participaban 25 alumnos que habían pagado la matrícula presencial de la University of Manitoba, y 2.200 estudiantes ajenos a la institución que participaron gratuitamente pero online. Todos los contenidos del curso estaban disponibles a través de notificaciones RSS, y los alumnos online podían participar a través de un gran número de herramientas sociales y colaborativas: blogs, debates en Moodle, reuniones en Second Life, etc.

Pero, es en 2011 cuando este tipo de cursos alcanzan su popularidad gracias al curso Introduction to Artificial Intelligence ofrecido por Sebastian Thrun y Peter Norvig en 
la Universidad de Stanford a través de una compañía startup llamada Know Labs (actualmente Udacity), en el que se matricularon más de 160.000 personas. Tras esta iniciativa empezaron a aparecer diferentes plataformas como Coursera o edX, que ofrecen MOOCs.

Según lo expuesto, este tipo de cursos son una evolución de la educación en abierto a través de internet y deben tener cuatro características fundamentales:

Deben ser masivos: es decir, deben ir dirigidos a un número ilimitado de alumnos. Estos cursos no tienen un número máximo de alumnos, si no que pueden apuntarse todos cuantos lo deseen.

Son cursos abiertos. Esta característica hace referencia a varios conceptos. En primer lugar son cursos en los que puede inscribirse cualquier persona, independientemente de su formación previa. No hay requisitos mínimos para inscribirse.

En segundo lugar el contenido es abierto; está disponible online.

En tercer lugar el concepto "abierto" hace referencia a que son gratuitos, es decir, que cualquier persona puede recibir formación impartida por los profesores de las universidades más exclusivas del mundo.

Son cursos online. Se imparten única y exclusivamente a través de internet por lo que son accesibles a alumnos desde cualquier parte del mundo.

Son cursos. Esto quiere decir que hay un profesor-tutor, unas fechas de inicio y fin y un sistema de evaluación.

Cada una de estas características aporta a este nuevo modelo educativo ventajas, pero también plantean muchos inconvenientes. A continuación realizaremos un profundo análisis de los mismos teniendo en cuenta sus ventajas y desventajas

\subsection{Fortalezas y debilidades como herramienta educativa}

Los MOOCsestán pensados para tener una audiencia masiva: cualquier individuo desde cualquier parte del mundo puede inscribirse. No hay un número máximo, ya que tal y como se ha apuntado anteriormente algunos han sobrepasado los 150.000 alumnos.

Una cifra así sólo puede ser gestionada de forma virtual. Sin embargo es muy complicado, por no decir imposible, proporcionar una atención personalizada como la que se ofrece en la enseñanza presencial. Aunque las TIC nos permiten distribuir información de manera masiva a través del mail o de los foros, la interacción personal y la conversación con el alumno se hace inviable e imposible en estos entornos.

Además de la comunicación, la masificación plantea otro importante problema: la evaluación. Aunque se están desarrollando diferentes programas informáticos diseñados para evaluar, corregir y proporcionar feedback al alumno, este problema limita mucho el tipo de ejercicios que pueden plantearse. 
La segunda característica de estos cursos es que son "online", esto implica que se realizan completamente a través de la red. Las ventajas de este sistema radican en que son accesibles para un número mayor de personas que por trabajo o cargas familiares no disponen de tiempo para desplazarse a un centro educativo a recibir formación. Además, son globales, esto es que cualquier persona de cualquier parte del mundo puede acceder a esos conocimientos. Cada alumno decide cuando y donde completar el curso, sin horarios y desde la comodidad de su domicilio.

Sin embargo la formación online también tiene sus desventajas, máxime si es gratuita. La tasa de abandono de los MOOCs, es decir, el número de alumnos que no llegan a completar la formación, es del 90\%. En el ya comentado curso Introduction to Artificial Inteligence, en el que se inscribieron inicialmente 160.000 estudiantes, sólo 23.000 completaron el curso (Hyman, 2012). Esto se debe a que ante la inexistencia de horarios y la "ausencia" del profesor a los alumnos les cuesta seguir una rutina formativa, más aún si estos cursos son gratuitos.

En tercer lugar hablamos de cursos abiertos, un concepto que implica múltiples acepciones:

Puede inscribirse cualquier persona, sin necesidad de credenciales y sin tener que demostrar un nivel mínimo de conocimientos para recibir la formación. Esto tiene una parte positiva y es que permite el acceso a cursos de élite a cualquier persona, sin embargo la parte negativa es que al no medir el nivel de los participantes ni requerir un perfil específico, la audiencia es heterogénea y se incrementa la tasa de abandono entre los que no poseen la base de conocimientos suficiente para seguir el curso.

Además el concepto “abierto" implica que el material del curso está completamente disponible a través de la red. Esto facilita el acceso de los usuarios a los contenidos pero plantea un problema importante con los derechos de autor de los materiales. Cuando un profesor se cambia de universidad ¿quién se queda con la propiedad de los materiales?

Por otro lado, "abierto" significa gratis. La idea de que los MOOCs sean gratuitos ha supuesto un gran hito en el ámbito educativo ya que supone democratizar la educación de élite. Este es un gran paso, más aún en los países subdesarrollados donde hay muy pocas personas capaces de costearse un curso en una universidad de élite, algo que puede contribuir enormemente al desarrollo ecónómico de estos países. Sin embargo la gratuidad de los MOOCs es también su mayor problema ¿cuál es el modelo de negocio? ¿Cómo pueden generar dinero para seguir creando materiales y pagar a los docentes? En este sentido y como veremos más adelante, no hay una solución clara.

Por último, son "cursos", el alumno recibe una formación, adquiere unas competencias y es evaluado por ello. El gran problema en este sentido es el de la evaluación y la certificación. Muchos alumnos se matriculan es estos cursos por el mero placer de aprender, sin embargo otros lo hacen porque necesitan adquirir determinadas competencias para mejorar en su trabajo o conseguir uno nuevo por lo que necesitan 
certificar sus conocimientos y la mayoría de los MOOCs no ofrecen un certificado que demuestre que el alumno ha realizado el curso; la evaluación es un tema muy complicado al tener una audiencia masiva.

\section{La revolución de la enseñanza superior}

Los MOOCs son un invento reciente pero han revolucionado el concepto de la educación superior y la formación a distancia. Pero, como comentábamos no todos los MOOC son iguales. De hecho, no existe unanimidad a la hora de afrontar su estudio. Para algunos autores estos cursos no son ni abiertos, ni masivos ni gratuitos (Zapata-Ros, 2013). Según Lisa Lane (2012) existen tres tipos de MOOC basados en:

Trabajo en red. La prioridad no reside en la adquisición de conocimientos o habilidades, sino en la construcción social del conocimiento, la conversación o el acceso a materiales en red. Serían los MOOCs originales defendidos por George Siemens y Stephen Downes.

Tareas. Se centran en que el estudiante complete una serie de trabajos propuestos para los que hay que desarrollar una serie de habilidades.

Contenidos. Son los cursos más mediáticos, aquellos promovidos por profesores de prestigio, con gran cantidad de alumnos inscritos y cierta orientación comercial. Se prima fundamentalmente la adquisición de conocimientos frente a la creación de una comunidad de usuarios, que se ve dificultada por la enorme cantidad de alumnos matriculados.

Pero la clasificación más extendida es la que divide estos cursos atendiendo a la teoría o corriente de aprendizaje correspondiente. Así, puede hablarse de cMOOC, para referirse a los conectivistas, y de xMOOC para los no conectivistas. Los cMOOCs son cursos gratuitos y verdaderamente accesibles que buscan el aprendizaje basándose fundamentalmente en la teoría conectivista del aprendizaje. Éste se consigue a través de la interacción con otros estudiantes (a través de blogs, feeds RSS) y son precisamente estas relaciones el mayor valor añadido de estos cursos. De hecho, en estos casos la certificación pasa a un segundo plano. Se trata de facilitar la creatividad y el trabajo colaborativo en red.

Por su parte, los xMOOCs, no conectivistas, se centran en el contenido que se ofrece únicamente a través de la página web y que permite gestionar grandes cantidades de alumnos insritos. En este caso, la colaboración es más rígida y la superación del curso se mide a través de la evaluación por test o trabajos. Coursera, Udacity y EdX son algunos ejemplos de xMOOCs. 


\subsection{Udacity}

Cuando los profesores de la Universidad de Stanford, Thrun y Sokolsky, crean Udacity en 2011 buscan explorar una nueva vía de la Educación Superior, reinventar la educación del siglo XXI haciendo que ésta fuera "accesible, asequible, agradable, y muy eficaz para el mundo". Así, los MOOCs permiten satisfacer un "derecho humano básico" con el que los estudiantes pueden adquirir conocimientos sobre diversas materias pero también avanzar en el desarrollo profesional de los trabajadores en activo (CARR, 2012). Se trata, en palabras de los creadores, de "construir un puente entre las habilidades del mundo real, una educación pertinente y el empleo".

You-dacity, es un juego palabras que combina la "audacia", la Universidad y el "tú", el estudiante. Según el declara el propio Thrun en su página web personal, Udacity significa "somos audaces, para ti, el estudiante".

Los resultados de Udacity en poco más de dos años son sorprendentes. Tiene registrados 160.000 alumnos de 190 países distintos. Esta plataforma sin ánimo de lucro ofrece en estos momentos casi una treintena de cursos clasificados en cinco categorías (Negocios, Ciencias de la Computación, Diseño, Matemáticas y Ciencia) $\mathrm{y}$ tres niveles de formación (Principiante, Intermedio y Avanzado). Al mismo tiempo, tiene asociada gracias a los servicios de meetup.com, un grupo de 611 comunidades de usuarios, los udacians, repartidas por 576 ciudades del mundo, con los que busca revitalizar las comunidades locales.

\subsection{EDX}

En mayo de 2012 los presidentes de la Universidad de Harvard y el Instituto Tecnológico de Massachusetts (MIT) anuncian la inversión de sesenta millones de dólares para la creación de edX (Kolovich, 2013). Un proyecto cuyo principio rector era mejorar la educación en el aula, no suplantarla. Hoy, poco más de un año después, esta plataforma está respaldada por un consorcio formado por veinte universidades de todo el mundo.

A pesar de considerarse como una plataforma sin ánimo de lucro y de que los cursos que ofrecen sean gratuitos, algunos de ellos cobran una tarifa por certificación. El dinero recaudado se reinvierte, según esta iniciativa, en la mejora de la plataforma. Según se explica en su propia página web, éstas pueden ser de tres tipos:

Honor Code: acredita sin coste alguno la superación del curso pero no refleja la identidad del alumno.

ID Verified Certificate: permite certificar que el alumno ha completado el curso, identificando al estudiante con su nombre y su foto. Este tipo de diploma tiene un coste asociado y sólo está disponible en una selección limitada de cursos.

Por último, el XSeries Certificate para aquellos estudiantes que haya superado una serie de cursos vinculados a una materia determinada. 


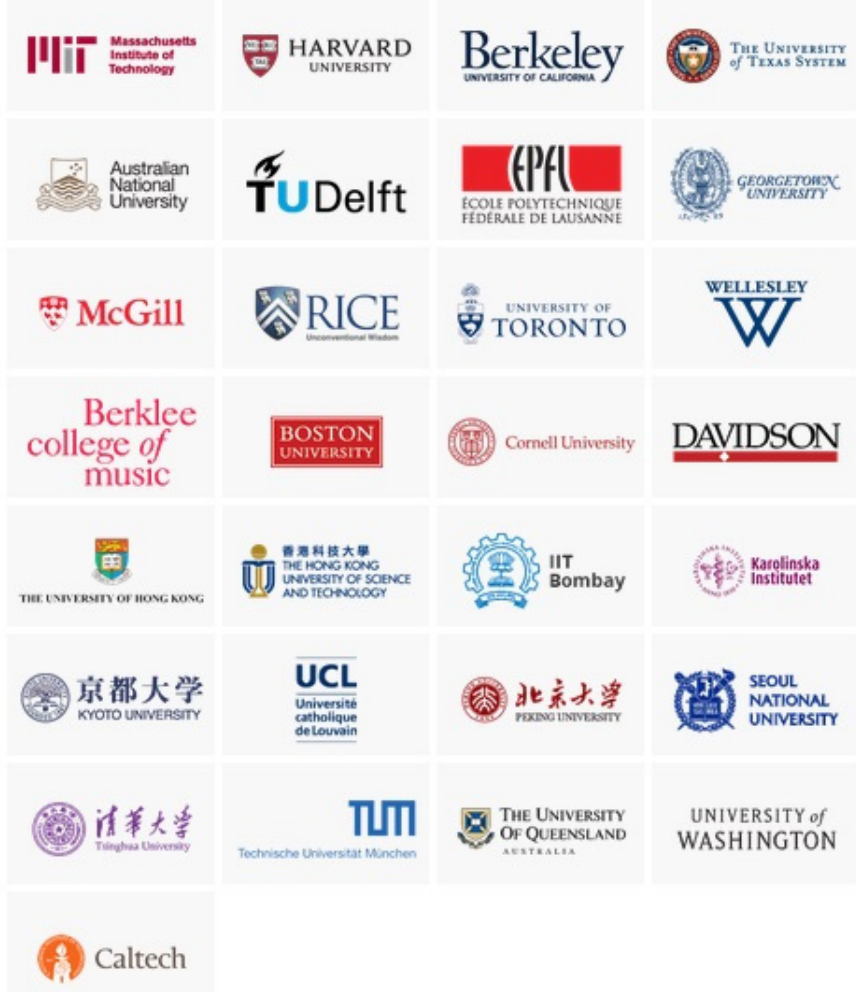

Consorcio de Universidades de edX. Fuente: www.edX.org

edX tiene disponibles 72 cursos divididos en nueve categorías principales: Derecho, Historia, Ciencia, Ingeniería, Negocios, Ciencias Sociales, Ciencias de la Computación, Salud Pública e Inteligencia Artificial.

\subsection{Coursera}

En términos cuantitativos, Coursera quizá sea la plataforma más importante. Cuenta actualmente con 447 cursos distribuidos en 23 categorías diferentes y, aunque se imparten dominantemente en inglés, también pueden cursarse en francés (12), español (12) o chino (10).

$\mathrm{Al}$ igual que las anteriores, la filosofía de este proyecto es permitir el acceso gratuito y mundial a la educación para "capacitar a las personas con educación que mejoren sus vidas, las vidas de sus familias y las comunidades en las que viven". Para ello, cuenta con el apoyo de una gran cantidad de partners universitarios de todo el mundo.

Las cifras conseguidas por esta iniciativa son increíbles. Según sus propias fuentes, a 14 de septiembre de 2013 Coursera cuenta con 17 millones de alumnos inscritos procedentes de 190 países en sus 440 cursos. Teniendo el curso más popular 240.000 
estudiantes. Ha conseguido generar 251.9 millones de minutos de clases y 590.000 hilos de discusión.

American Museum of Natural His.
Berklee College of Music
Brown University
California Institute of Technology
California Institute of the Arts
Case Western Reserve University
Columbia University
Commonwealth Education Trust
Curtis Institute of Music
Duke University
École Polytechnique
École Polytechnique Fédérale de
Emory University
Exploratorium
Georgia Institute of Technology
Hebrew University ofJerusalem
IE Business School
Icahn School of Medicine at Mou
Johns Hopkins University
Ludwig-Maximilians-Universität ..
Match Teacher Residency
National Taiwan University
National University of Singapore
New Teacher Center
Northwestern University
Peking University New

Pennsylvania State University
Princeton University
Relay Graduate School of Educa...
Rice University
Rutgers University
Sapienza University of Rome
Stanford University
Technical University of Denmark..
Technion - Israel Institute of Tech...
Technische Universität München ...
Tecnológico de Monterrey
Tel Aviv University
The Chinese University of Hong K...
The Hong Kong University of Scie...
The Museum of Modern Art
The Ohio State University
The University of British Columbia
The University of Chicago
The University of Edinburgh
The University of North Carolina ...
The University of Tokyo
Universidad Nacional Autónoma ...
Universitat Autònoma de Barcelo...
Universiteit Leiden
University of Alberta NEW
University of Amsterdam NEW

\author{
University of California, Irvine \\ University of California, San Diego \\ University of California, San Fran. \\ University of California, Santa $\mathrm{Cr}$ \\ University of Colorado Boulder \\ University of Copenhagen \\ University of Florida \\ University of Geneva \\ University of Illinois at Urbana-C. \\ University of London Internationa \\ University of Maryland, College \\ University of Melbourne \\ University of Michigan \\ University of Minnesota \\ University of Pennsylvania \\ University of Pittsburgh \\ University of Rochester \\ University of Toronto \\ University of Virginia \\ University of Washington \\ University of Wisconsin-Madison \\ University of Zurich \\ Vanderbilt University \\ Wesleyan University \\ Yale University
}

Consorcio de Universidades de Coursera. Fuente: www.coursera.org

Pero, Coursera está trabajando para ir más allá y, partiendo de este modelo de educación masiva abierta, ofrece a las empresas una manera de colaborar en la formación continua de sus trabajadores. De este modo, las empresas que así lo deseen pueden poner a disposición de sus recursos humanos una amplia variedad de cursos que, a cambio de una cantidad económica (una esponsorización), la plataforma se encargará de validar y certificar convenientemente. Se trata, por tanto, de un modelo de negocio alternativo que ayuda al mantenimiento de la iniciativa gratuita. 


\section{Otras iniciativas: Europa y España}

La Unión Europea, concienciada en la importancia del conocimiento como derecho pero también como modo para salir de la crisis, no ha querido quedar atrás. Coordinada por la EADN (acrónimo en inglés para Asociación Europea de Universidades a Distancia) y respaldada por la Comisión Europea, el objetivo de OpenupED es acercar una "educación que refleje los valores europeos, tales como la equidad, la calidad y la diversidad". Así, busca ofrecer un modelo de universidad abierta con materiales de auto-aprendizaje de alta calidad.

Cuenta con once universidades repartidas por diez países distintos: Francia, Italia, Lituania, Países Bajos, Portugal, Eslovaquia, España, Gran Bretaña, Rusia, Turquía e Israel. Inicialmente se lanza con 40 cursos disponibles en doce lenguas distintas y en estos momentos tiene acumulados un total de 71 cursos.

Por último, tenemos que hablar de Miriadax. Bajo el paraguas de Universia -una red de 1.242 universidades iberoamericanas-, y el apoyo tecnológico de Telefónica Learning Services -compañía especializada en ofrecer soluciones integrales de aprendizaje online-, constituye la apuesta española más importante dirigida a todo el mercado hispanohablante. Tiene un doble propósito: apoyar a los estudiantes universitarios para el perfeccionamiento de conocimientos durante su carrera y colaborar en la mejora del perfil profesional de aquellos que ya están trabajando. Para ello, ofrece cursos diseñados y organizados por veinte universidades en distintas áreas de conocimiento.

La estructura y funcionamiento es similar a las expuestas anteriormente pero profundiza en la dimensión más social de este tipo de educación. Los cursos cuentan con video-lecciones que se completan con actividades obligatorias y optativas. Se ofrecen dos tipos de certificaciones a los estudiantes:

Certificado de participación, cuando se hayan completado el $75 \%$ de las actividades obligatorias.

Certificado de superación al entregar el 100\% de las tareas encomendadas.

Además, la plataforma entrega badges, una insignia virtual "que refleja una habilidad o logro conseguido". En este caso, por cada curso conseguido.

Otro de los elementos más significativos es el Karma o la valoración de las aportaciones de los estudiantes. Cuanto más y mejor valoradas sean estas aportaciones a través de las herramientas colaborativas (foros, blogs, etc.), más karma irá recibiendo el alumno. De este modo, "se eleva la calidad de las aportaciones de los usuarios, se evitan comportamientos poco apropiados, favoreciendo el respeto y mejorando la experiencia de aprendizaje entre todos". 


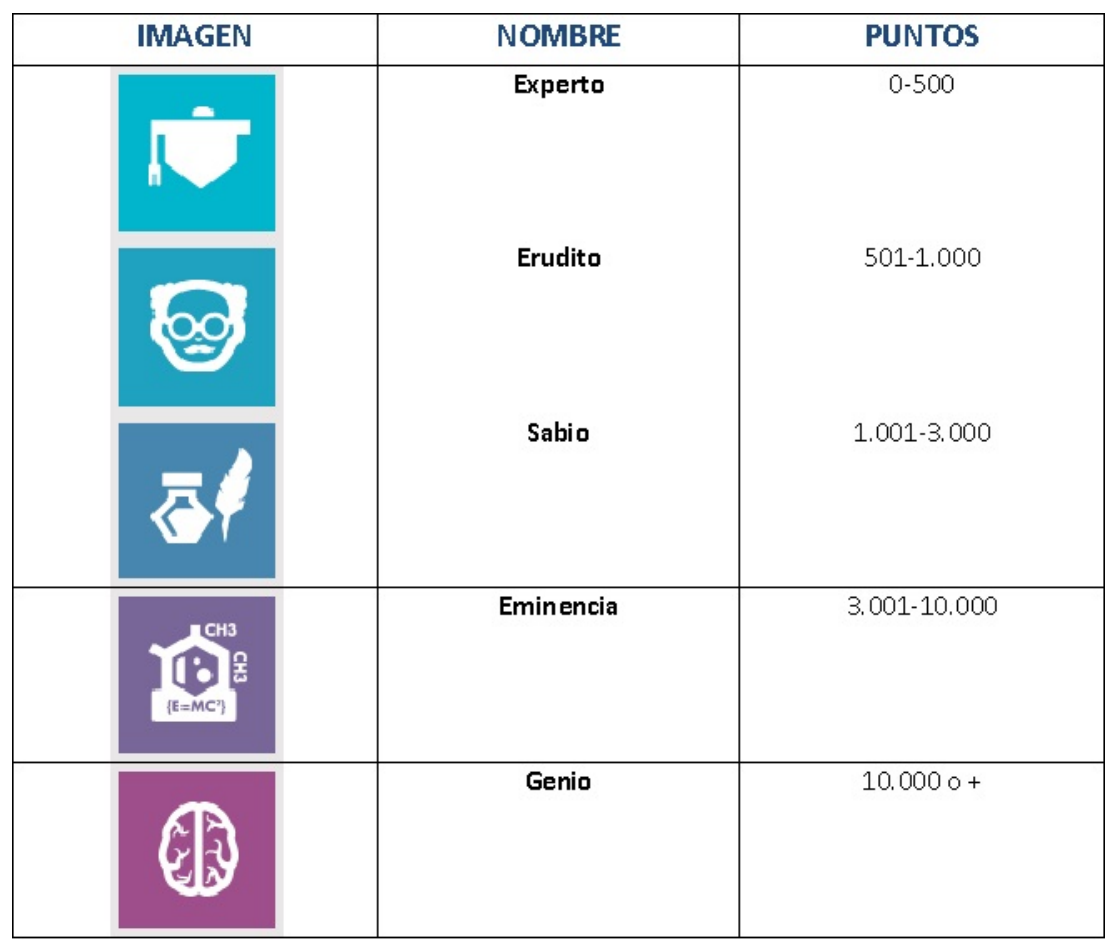

Tipos de Karma. Fuente: www. miriadax.net

\section{5. ¿Un nuevo modelo de negocio?}

Muchos son los argumentos a favor y en contra de este tipo de cursos online. Si bien permiten gestionar un gran número de alumnos mediante la implantación de las TIC y mejorar así la productividad, también encontramos voces críticas en contra de los MOOCs. Principalmente por la mencionada tasa de abandono pero, ante todo, por la imposibilidad de sustituir al profesor y su interacción con el alumno. Planteadas ya estas cuestiones debe ponerse en relieve un elemento fundamental para la supervivencia de estas fórmulas académicas: la necesaria definición de un modelo de negocio. Han sido las universidades más prestigiosas del mundo las que han iniciado y liderado esta nueva fórmula de educación, éstas se cuestionan ahora cómo rentabilizar los crecientes gastos asociados (Cavanagh, 2013).

Como comentábamos, esta fórmula de Social Learning tiene como una de sus premisas esenciales la gratuidad de la oferta formativa. El desafío se encuentra actualmente en combinar una oferta atractiva de cursos, que sirva a su vez para retroalimentar la imagen de la institución, con un sistema de financiación que permita cumplir con los objetivos anteriores. 
Algunas iniciativas han apostado por implantar el cobro de una cantidad simbólica en concepto de matrícula. Un mecanismo que puede mejorar notablemente la rentabilidad económica. Con sólo cobrar un euro a cada alumno, un MOOC podría recaudar miles de euros si tenemos en cuenta las increíbles cifras de alumnos matriculados que se han expuesto.

Otras plataformas de MOOCs son partidarias de ofrecer formación inicialmente accesible de manera gratuita pero cobrar una tarifa económica en caso de que el alumno quiera un certificado que acredite los conocimientos y/o competencias evidenciadas a lo largo del curso. De este modo, el alumno podría incorporar este certificado a su acreditación y con su participación económica se sostendría el sistema para seguir ofreciendo formación gratuita. Este mecanismo permitiría la pervivencia de del espíritu original de los MOOCs.

No obstante, debemos resaltar una última vía para el desarrollo económico de estos cursos masivos online y es la que vincula a las instituciones educativas con el mercado profesional. Consistiría en ofrecer a las empresas este tipo de cursos, tal y como hace Coursera, como base de su catálogo de formación continua para sus trabajadores. De esta manera, universidad, empresa y trabajadores ganarían mediante esta alianza. La universidad obtendría reconocimiento y sustento económico del mercado profesional; las empresas mejorarían su plan de formación interna a un coste asequible y los trabajadores tendrían una formación especializada y certificada por instituciones de reconocido prestigio.

\section{Conclusiones}

La reciente irrupción de los MOOCs en el ámbito educativo supone la llegada de la democratización en la enseñanza de la mano de las Tecnologías de la Información y la Comunicación (TIC). 2012 ha sido el año de la consolidación de este tipo de cursos. Bajo esta denominación se engloban una gran cantidad de iniciativas, de las que aquí se han expuesto las más significativas, con diferentes filosofías de trabajo. Más allá de las diferencias que separan, los MOOCs se han convertido en un tipo de formación muy atractiva para todo tipo de alumnos. La marca asociada a las grandes instituciones universitarias, el prestigio de los profesores o el contenido especializado conforman los aspectos más atractivos de esta modalidad de enseñanza.

Pero, pasada esta enorme expansión inicial cabe plantearse el futuro de este tipo de formación. Como hemos mencionado, quizá sea el problema de la financiación el tema más recurrido. Es necesario definir un modelo de negocio que permita asegurar la viabilidad financiera de este tipo de cursos. Es imposible que las universidades sigan costeando a fondo perdido estas iniciativas. No obstante, este sistema de financiación no debe ir en contra de la propia naturaleza de los MOOCs, limitando el acceso a esta formación a aquellos estudiantes con mayores recursos económicos. 


\section{Bibliografía}

Artículos en revistas de papel

CARR, NICHOLAS (2012). "The crisis on Higher Education". En MIT Technology Review, vol. $115, \mathrm{n}^{\mathrm{o}} 6$.

CAVANAGH, SEAN (2013). "MOOCs provider targets teacher education. En Education Week, Mayo 2013.

HYMAN, PAUL (2012). "In the year of disruptive education". En Communications of the ACM, vol. 55, $\mathrm{n}^{\circ} 12$.

SEELY, JOHN (2008). "Foreword: Creating a Culture of Learning". En IIYOSHI, TORU, VIJAY KUMAR: Opening Up Education: The Collective Advancement of Education Through Open Technology, Open Content, and Open Knowledge, Creative Commons edition, ed. and (Princeton, New Jersey: Carnegie Foundation for the Advancement of Teaching, 2008), xi-xvii; and Brown and Adler, "Minds on Fire."

Artículos en publicaciones web

ATKINS, DANIEL; SEELY, JOHN, HAMMON, ALLEN (2007): A Review of the Open Educational Resources (OER) Movement: Achievements, Challenges, and New Opportunities, Report to The William and Flora Hewlett Foundation. Disponible en: www.hewlett.org/uploads/files/Hewlett_OER_report.pdf. [09-09-2013].

KOLOVICH, STEVE (2013): The Professors Who Make the MOOCs. Disponible en http://chronicle.com/article/The-Professors-Behind-the-MOOC/137905/\#id=overview. [22-09-2013].

LANE, LISA (2012). Three kinds of MOOCs. Disponible en: http://lisahistory.net/ wordpress/2012/08/three-kinds-of-moocs/. [22-09-2013 de septiembre de 2013]

LÓPEZ SANDINO, JORGE (2012). MOOC: ¿Ocaso del modelo de negocio tradicional en e-learning? Disponible en http://ojulearning.es/2012/08/mooc-ocaso-del-modelo-de-negocio-tradicional-en-e-learning/ [22-09-2013]

MOOC.ES .cMoox vs. XMooc. http://www.mooc.es/cmoox-vs-xmooc/ [22-09-2013]

ZAPATA-ROS, MIGUEL (2013). MOOCs, una visión crítica. El valor no está en el ejemplar. Disponible en http://eprints.rclis.org/18452/1/MOOC_critica_Elis.pdf. [11-06-2013]

\section{Las autoras}

Raquel Vinader Segura. Licenciada en Comunicación Audiovisual por la Universidad Complutense de Madrid y Máster en Dirección de Empresa por la Carlos III de Madrid. Doctora en Ciencias de la Información por la UCM. Actualmente es profe- 
sora de Empresa Audiovisual en el CES Felipe II (UCM). Miembro del grupo de Investigación FONTA (Formación en Nuevas Tecnologías del Audiovisual).

Natalia Abuín Vences. Licenciada en Periodismo por la Universidad de Santiago de Compostela. Doctora en Ciencias de la Información por la Universidad Complutense de Madrid. Actualmente es profesora de Cultura de las Organizaciones en el Departamento de Comunicación Audiovisual y Publicidad 1 de la UCM. Coordinadora del grupo de Investigación FONTA (Formación en Nuevas Tecnologías del Audiovisual). 